\title{
Influência do aditivo oxibiodegradante orgânico na degradação de um biocompósito e na fertilidade do solo
}

\section{Cristiano Cunha Costa}

Doutorado em Ciência e Engenharia de Materiais pela Universidade Federal de Sergipe, Brasil

Docente Externo da Universidade Federal de Sergipe , Brasil

\section{José Airon da Silva}

Doutor em Agronomia (Ciência do Solo) pela Universidade Federal Rural de Pernambuco / UFRPE

\section{Luís Eduardo Almeida}

Orientador de Mestrado | Orientador de Doutorado

Doutorado em Físico Química pelo Instituto de Química de São Carlos, Brasil(2000)

Trabalha na Universidade Federal de Sergipe, Brasil

\section{RESUMO}

Diante da dificuldade de degradação do material plástico, alguns aditivos são usados para estimular esse processo. $O$ objetivo deste trabalho foi estudar a biodegradação de um compósito de polietileno e casca de arroz com um aditivo oxibiodegradante orgânico. Para isso, os compósitos foram submetidos a um envelhecimento acelerado e, em seguida, levados ao ensaio de biodegradação. Realizou-se uma análise da fertilidade do solo simulado, para verificar o efeito do aditivo nas suas propriedades químicas, após a sua ação. O ensaio de líquido penetrante sugere que a superfície do compósito $\mathrm{PEAD} / \mathrm{CA} / \mathrm{EG}$ resultou mais degradada, o que favoreceu a ação de microrganismos com atividade de biodegradação do polietileno, como o Aspergillus níger, Penicillium spp., Trichoderma spp., Rhizopus spp. O aditivo oxibiodegradante orgânico não produziu efeitos nas propriedades químicas do solo, pois não há metais pesados em sua composição; assim, não causa danos ao meio ambiente nem à saúde.

Palavras-chave: Oxibiodegradante orgânico. Biodegradação. Metais pesados. Fertilidade do solo. 


\title{
Influence of organic oxo-biodegradation additive in degradation of a composite and soil fertility
}

\author{
Influencia del aditivo con función oxobiodegradable orgánico en la \\ degradación de un biocompuesto y en la fertilidad del suelo
}

\begin{abstract}
Given the difficulty of degradation of the plastic material, some additives are used to stimulate this process. The objective of this work was to study the biodegradation of a composite of polyethylene and rice husk with an organic oxo-biodegradant additive. For this, the composites were subjected to accelerated aging and then taken to the biodegradation test. A simulated soil fertility analysis was performed to verify the effect of the additive on its chemical properties after its action. The penetrating liquid test suggests that the surface of the HDPE / CA / EG composite was more degraded, which favored the action of microorganisms with polyethylene biodegradation activity, such as Aspergillus niger, Penicillium spp., Trichoderma spp., Rhizopus spp. The organic oxo-biodegradant additive had no effect on soil chemical properties, as there are no heavy metals in its composition; thus, it does not cause damage to the environment or health.
\end{abstract}

Keywords: Organic oxo-biodegradant. Biodegradation. Heavy metals. Soil fertility.

\section{RESUMEN}

Ante la dificultad de la degradación del plástico, algunos aditivos son utilizados para estimular ese proceso. El objetivo de este trabajo fue estudiar la biodegradación de un compuesto de polietileno y cascarilla de arroz con un aditivo con función oxobiodegradable, orgánico. Para ello, los compuestos fueron sometidos a un envejecimiento acelerado y, en seguida, llevados al ensayo de biodegradación. Se realizó un análisis de fertilidad del suelo simulado, para verificar posibles efectos del aditivo sobre sus propiedades químicas, luego de su acción. La prueba de líquido penetrante sugiere que la superficie del compuesto PEAD/CA/EG resultó más degradada, lo que favoreció la acción de microorganismos con actividad de biodegradación del polietileno, como el Aspergillus níger, Penicillium spp., Trichoderma spp., Rhizopus spp. El aditivo con función oxobiodegradable orgánico no produjo efectos sobre las propiedades químicas del suelo, una vez que no hay metales pesados en su composición; por ello, no causa daño al medio ambiente ni a la salud.

Palabras-clave: Aditivo con función oxobiodegradable orgánico. Biodegradación. Metales pesados. Fertilidad del suelo.

\section{INTRODUÇÃO}

Os plásticos comumente usados, entre eles o polietileno, são resistentes à degradação biológica, o que resulta na sua permanência no meio ambiente por muito tempo e cria desafios quanto à gestão desse tipo de resíduo (GOMEZ et al., 2013). Tal fato ocorre devido à sua característica de hidroficidade e resistência à hidrólise, e por esta razão não podem biodegradar, necessitando da ação de fatores abióticos para favorecer os processos de degradação (ARRÁEZ et al., 2018).

Alguns ramos da engenharia têm procurado novas tecnologias no que se refere a alternativas para a redução do acúmulo de resíduos poliméricos no meio ambiente, através 
do desenvolvimento de materiais oxibiodegradáveis (GROSS; KALRA, 2002; BOTELHO et al., 2004).

Os materiais oxibiodegradáveis pertencem à categoria das poliolefinas tradicionais (polietileno, por exemplo) às quais são adicionados catalisadores que aceleram a oxidação química das cadeias poliméricas, denominados agentes oxibiodegradantes (ARRÁEZ et al., 2018).

Os oxibiodegradantes apresentam uma combinação iônica de metais como, por exemplo, manganês, cobre, ferro, cobalto, entre outros, que favorece o processo de catalisação na decomposição dos peróxidos associados com a quebra da cadeia do polietileno e, consequentemente, conduz ao processo de biodegradação (BONHOMME et al., 2003; YAMADA-ONODERA et al., 2001; KOUTNY et al., 2006).

A adição de oxibiodegradantes na cadeia de polietileno favorece o seu processo de degradação devido à formação de radicais livres os quais reagem com o oxigênio, resultando em peróxidos e hidroperóxidos que, por sua vez, atuam como iniciadores da auto-oxidação do polietileno e participam dos mecanismos de oxidação, levando a reações consequentes, via radical livre, e à oxidação do polímero (KHABBAZ et al., 1999). Deste modo, a presença de oxibiodegradantes tem favorecido a degradação abiótica, uma vez que facilita a quebra da cadeia do polietileno, em um primeiro momento, resultando em cadeias menores as quais serão consumidas pelos microrganismos na etapa de biodegradação (CORTI et al., 2010; JAKUBOWICZ, 2003).

$\mathrm{Na}$ literatura, há estudos que relatam o comportamento da degradação do polietileno com aditivos oxibiodegradantes que possuem metais pesados na sua composição como, por exemplo, Manganês (ABRUSCI et al., 2013; KIRYAKOVA et al., 2016; BENÍTEZ et al., 2013; KONDURI et al., 2011; MARYUDI et al., 2012; MARYUDI et al., 2013; JAKUBOWICZ et al., 2011), Cobalto (ABRUSCI et al., 2013; KIRYAKOVA et al., 2016; SAMAL et al, 2014; KONDURI et al., 2011; FOCKE et al., 2011), Titânio (YANG et al., 2006; MANANGAN et al., 2010; KONDURI et al., 2011), Cobre (FOCKE et al., 2011), entre outros.

Entretanto, tais aditivos oxibiodegradantes, que possuem íons de metais na sua composição, causam sérios danos à saúde humana e impactos negativos ao meio ambiente, dependendo da sua concentração, uma vez que possuem efeito acumulativo na cadeia trófica (MAGNA et al., 2014). 
Dentro desse contexto, a adição de oxibiodegradantes orgânicos tem a função de promover/acelerar o processo de oxidação química das cadeias poliméricas, e essas reações podem ser desencadeadas após exposição à luz e/ou calor, permitindo a aceleração da biodegradação do compósito e, consequentemente, reduzindo a sua vida útil (SAMAL et al., 2014; MONTAGNA et al., 2014). Deste modo, o processo de degradação do polietileno a partir de oxibiodegradantes orgânicos não causa impactos negativos ao meio ambiente, pois não resultam em íons de metais pesados na natureza.

Este trabalho teve o objetivo de estudar a influência de um aditivo oxibiodegradante orgânico na biodegradação de um compósito e na fertilidade do solo, no sentido de buscar alternativas para o uso de novos produtos, aliados a uma minimização do prejuízo à natureza. Os compósitos foram submetidos a degradação abiótica por envelhecimento acelerado, por radiação ultravioleta (UV) e, em seguida, foram submetidos à biodegradação por um período de 180 dias. As análises da superfície dos compósitos por líquido penetrante, a identificação dos microrganismos degradadores e a fertilidade do solo após a biodegradação foram avaliados e os resultados foram apresentados neste trabalho.

\section{MATERIAIS E MÉTODOS}

\section{Reagentes}

Como matriz polimérica foi utilizado o polietileno de alta densidade (PEAD) fornecido pela Braskem (código IA59U3). O aditivo oxibiodegradante orgânico usado foi o EG15 fornecido pela TIV Plásticos (São Paulo/Brasil). A casca de arroz (CA) micronizada (com distribuição granulométrica entre 275 e 512,5 $\mu \mathrm{m}$ ) foi fornecida pela Cerealista Polisul (Pelotas/Brasil).

\section{Preparação dos compósitos}

Antes da extrusão, devido à higroscopicidade, a casca de arroz foi seca em estufa de circulação de $\operatorname{ar}\left(\right.$ SOLAB Científica, SL 102/480), a uma temperatura de $120^{\circ} \mathrm{C}$ por 4 horas, aproximadamente. Em seguida, todos os componentes foram misturados para a homogeneização. A extrusão ocorreu em extrusora dupla rosca co-rotacional (Imacon, 
DRC 30:40 IF). Depois, o material extrudado foi transformado em pellets, os quais foram usados na injeção dos corpos de prova. As composições foram as seguintes: PEAD (100\% de polietileno de alta densidade); PEAD/EG (95\% PEAD e 5\% de EG); PEAD/CA (80\% PEAD e $20 \%$ de casca de arroz) e PEAD/CA/EG (75\% de PEAD, 20\% de casca de $5 \%$ de EG).

Antes do processo de injeção, o material extrudado foi seco em estufa de circulação de ar, a uma temperatura de $120^{\circ} \mathrm{C}$ por 4 horas. Em seguida, os compósitos foram moldados em uma injetora (Battenfeld, modelo HM 45/210), de acordo com a norma ISO 527-93.

Para o envelhecimento acelerado, os compósitos foram acondicionados em câmara de envelhecimento UV do fabricante Bass, modelo UUV/2011. O processo foi realizado conforme adaptação das normas da ASTM G154-06 e ASTM G151-10, utilizando radiação UVA-340 com ciclos de exposição de 8 horas ao UVA a $60^{\circ} \mathrm{C}$ e 4 horas de condensação a $50^{\circ} \mathrm{C}$, perfazendo 90 ciclos, durante um período de 2.160 horas.

Em seguida, os compósitos foram submetidos ao ensaio de biodegradação em solo simulado conforme adaptações da norma ASTM G160-12, sendo avaliados após 90 e 180 dias.

Depois do ensaio de biodegradação, a superfície dos compósitos foi analisada pela técnica de líquido penetrante (LP). Para isso, utilizou-se uma adaptação da norma N-1596 recomendada pela Petrobrás (PETROBRAS, 2003). O líquido penetrante foi aplicado nos corpos de prova, e aguardou-se até completa secagem. Em seguida, foram lavados em água corrente e secados para a posterior aplicação do líquido revelador. O líquido penetrante (VP 30) e o revelador (D 70) são da Metal Check indicados para ensaios não destrutivos.

Para verificar a ação do aditivo oxibiodegradante orgânico, foram realizadas análises de microbiologia e de fertilidade do solo após a biodegradação. Para a análise microbiológica do solo, os corpos de prova submetidos ao ensaio de biodegradação foram lavados com água destilada. Em seguida, deu-se início a metodologias de identificação e isolamento das estruturas biológicas microscópicas.

O líquido fruto do processo de lavagem dos corpos de prova foi centrifugado a 1500 rpm por 10 min em uma centrífuga da marca Fanem-baby, modelo 206BL.

O sobrenadante foi descartado e $1 \mathrm{~mL}$ de sedimento foi inoculado com uma espátula de platina sobre Ágar Sabouraud e incubou-se a $25^{\circ} \mathrm{C}$ durante sete dias num incubador de cultura bacteriológica, modelo 502, Orion, Fanem. Observou-se diariamente o crescimento 
e estudou-se a macro e micromorfologia dos fungos. Com auxílio de um microscópio óptico da marca Zeiss, modelo Axiostar, foi possível identificar estruturas de helmintos, protozoários, fungos e bactérias existentes no solo simulado. Foi usada, como referência, a literatura específica (BARNETT; HUBTER, 1972; LACAZ et al., 1998) e cópias de táxons da micoteca do Laboratório de Micologia Médica da Universidade de Caxias do Sul do Rio Grande do Sul.

A avaliação da fertilidade do solo foi realizada no laboratório de solos do Departamento de Engenharia Agronômica da Universidade Federal de Sergipe, segundo metodologia sugerida pela Empresa Brasileira de Pecuária e Agricultura (EMBRAPA, 2009), sendo possível identificar potencial hidrogeniônico $(\mathrm{pH})$ e concentrações de íons de minerais como cálcio-magnésio $\left(\mathrm{Ca}^{2+}+\mathrm{Mg}^{2+}\right)$, cálcio $\left(\mathrm{Ca}^{2+}\right)$, magnésio $\left(\mathrm{Mg}^{2+}\right)$, potássio $\left(\mathrm{K}^{+}\right)$, sódio $\left(\mathrm{Na}^{+}\right)$, alumínio $\left(\mathrm{Al}^{3+}\right), \mathrm{H}+\mathrm{Al}$, fósforo $(\mathrm{P})$, e quantidade de matéria orgânica $(\mathrm{MO})$. Desta maneira, tais informações permitiram saber se o aditivo oxibiodegradante orgânico influenciou na qualidade da fertilidade do solo.

\section{RESULTADOS E DISCUSSÃO}

Na figura 1 estão as fotografias da superfície dos compósitos após o envelhecimento acelerado e ensaio de biodegradação aos 90 e 180 dias. Verifica-se que os compósitos PEAD e PEAD/EG não apresentaram qualquer indício de degradação superficial na sua condição natural ou após envelhecimento acelerado por radiação UV aos 90 e 180 dias de biodegradação, pois suas superfícies apresentam uma cloração branca, devido à fixação do líquido penetrante. 
Figura 1: Ensaio por líquido penetrante dos compósitos após envelhecimento acelerado e biodegradação aos 90 e 180 dias.

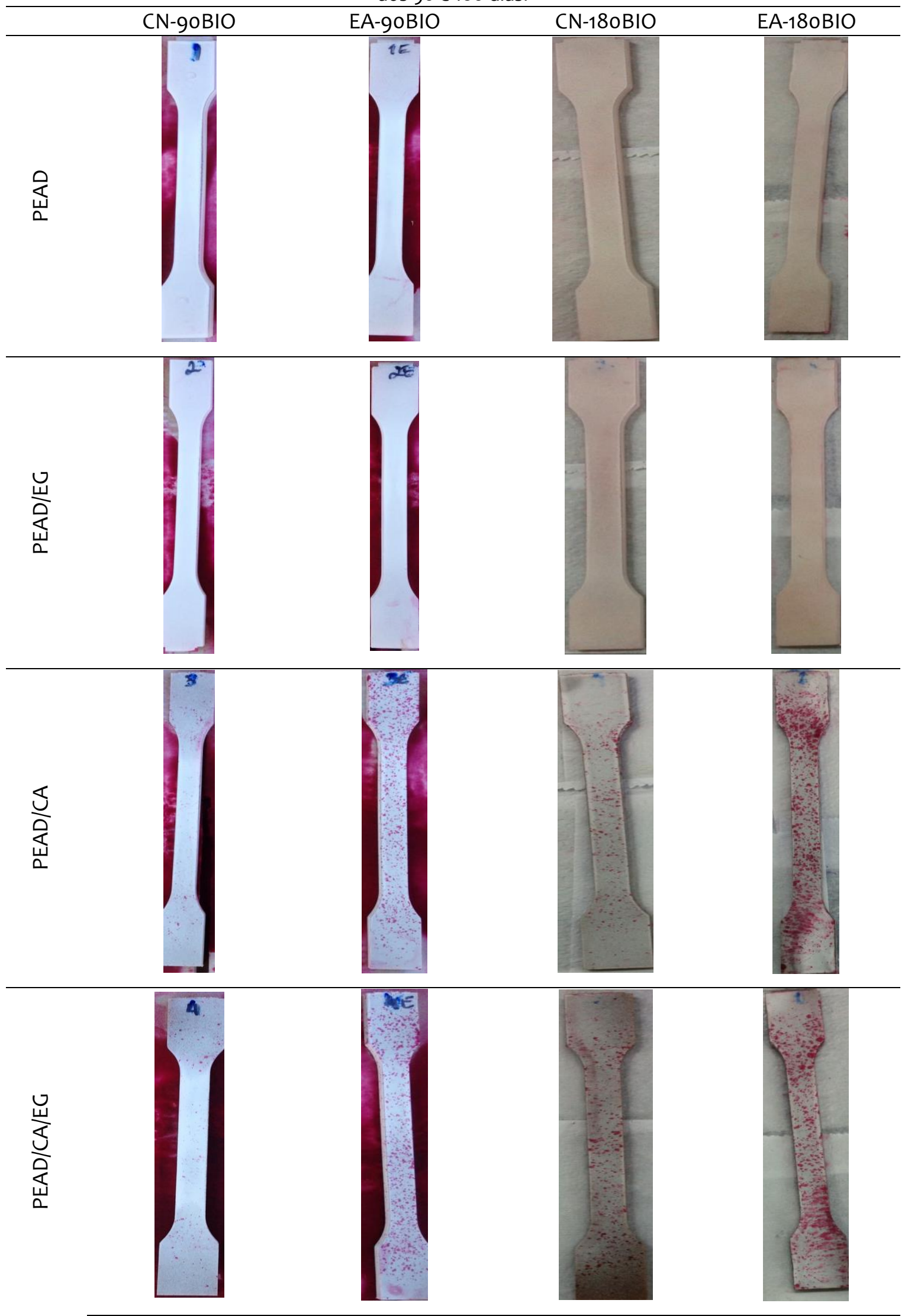


Por outro lado, nota-se que os compósitos que possuem as partículas de casca de arroz e aditivo oxibiodegradante (composições PEAD/CA e PEAD/CA/EG) apresentaram maior degradação superficial após o envelhecimento acelerado, a qual se intensificou após o ensaio de biodegradação em solo simulado aos 90 e 180 dias, apresentando buracos e crateras, uma vez que para esses compósitos há uma concentração maior de manchas vermelhas por conta da ação do líquido revelador.

Em estudo sobre a fotodegradação de compósitos de madeira e polietileno de alta densidade, Stark e Matuana (2006) concluíram que a degradação das partículas de madeira ocorre, principalmente, a partir da deterioração da lignina, pois é uma região rica em celulose, que se torna mais sensível à presença de umidade e, consequentemente, as paredes celulares da madeira incham, facilitando a penetração da luz e favorecendo uma maior degradação.

A análise microbiológica permitiu observar que os compósitos foram degradados por algumas colônias de fungos e bactérias, tornando possível a sua identificação a partir da macromorfologia (figura 2).

Figura 2: Colônias de microrganismos do solo simulado 7 dias após inoculação: (a) amostra de solo com 90 dias de biodegradação. (b) amostra de solo com 180 dias de biodegradação.
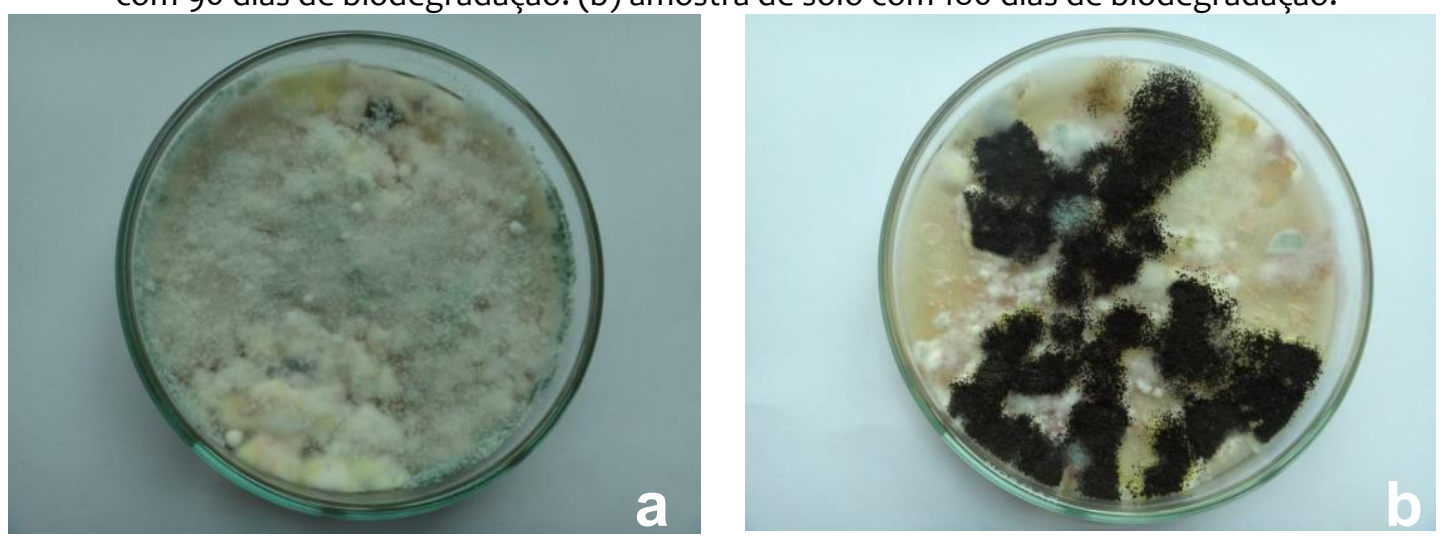

Em todas as amostras de solo simulado para 90 e 180 dias de biodegradação, foram identificadas as mesmas estruturas: colônias de fungos filamentosos negros de Aspergillus níger, colônias de fungos filamentosos verde-musgo de Penicillium spp., colônias de fungos filamentosos verdes de Trichoderma spp., colônias de fungos filamentosos cinza de Rhizopus spp. e colônias de bactérias e leveduras cremosas brancas não identificadas. 
Tais espécies fúngicas são relatadas, na literatura, como microrganismos com atividade de biodegradação do polietileno, apresentando resultados satisfatórios na colonização e degradação superficial da matriz polimérica. Assim, podem-se destacar trabalhos realizados com Aspergillus niger (KYAW et al., 2012; GOMES et al., 2014; DEVI et al., 2015, SHABANI et al., 2015), Penicillium spp. (KYAW et al., 2012; GOMES et al., 2014), Trichoderma spp. e Rhizopus ssp. (GOMES et al., 2014), entre outros.

Tal fenômeno é possível pelo fato de tais microrganismos produzirem enzimas do tipo lipase, por exemplo, que atuam especificamente na matriz polimérica de polietileno, degradando-a em fragmentos de cadeias menores do tipo de oligômeros, dímeros e monômeros, que servem de nutrientes para os fungos e bactérias (TOKIWA et al., 2009; BHARDWAJ et al, 2012).

A presença microrganismos é importante, na etapa de degradação biótica de compósitos de polietileno com partículas de casca de arroz e com a presença de oxibiodegradantes, pois tal ação é possível porque há a formação de um biofilme, como pode ser visto na superfície do compósito PEAD/CA/EG (figura 3), que comprova que está ocorrendo a degradação do biocompósito conforme já abordado na literatura (Devi et al., 2015; Mathur et al., 2011).

Figura 3: Micrografias dos compósitos com a "formação de biofilmes" após 180 dias de biodegradação: (a) Compósito PEAD/CA; (b) Compósito PEAD/CA/EG.
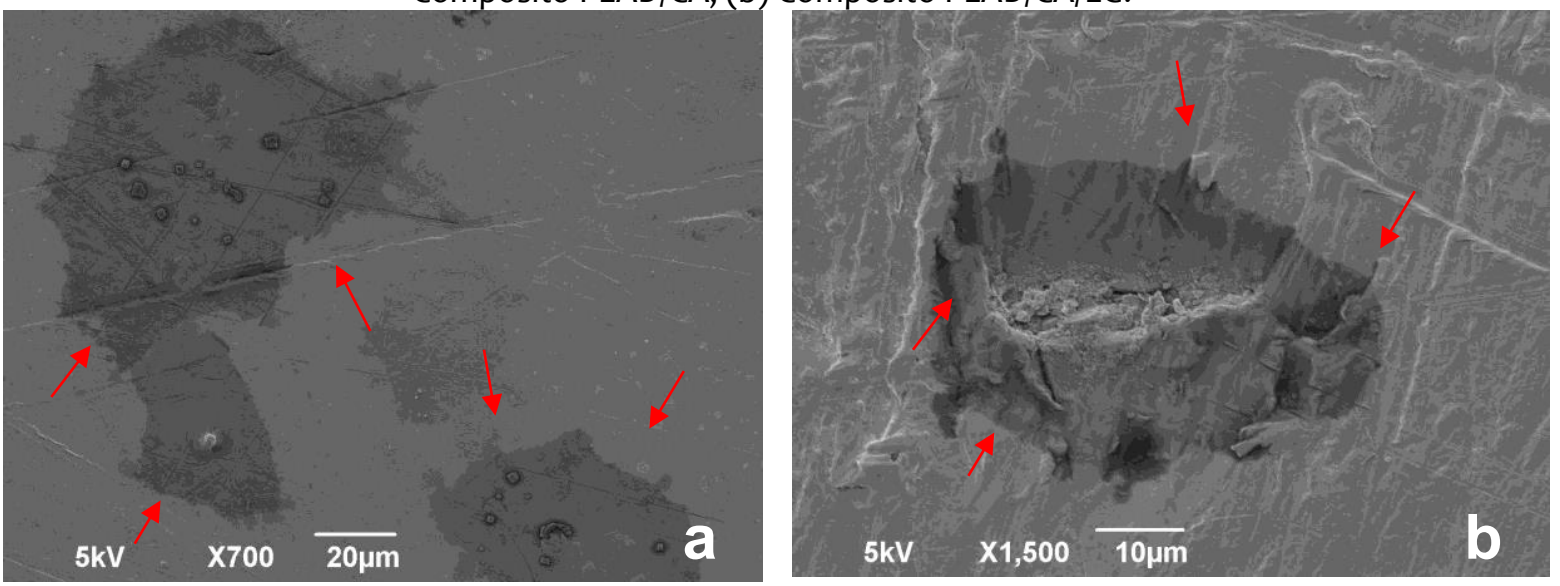

A análise da fertilidade do solo teve o objetivo de verificar alguma alteração nas propriedades químicas do solo devido ao ensaio de biodegradação em solo simulado, por conta da ação do oxibiodegradante orgânico. 
O resultado da análise da fertilidade do solo simulado, antes e após o ensaio de biodegradação, está apresentado na tabela 1.

Tabela 1: Resultado da análise da fertilidade do solo simulado antes e depois do ensaio de biodegradação.

\begin{tabular}{|c|c|c|c|c|c|c|c|c|c|c|c|c|}
\hline \multirow{2}{*}{ Amostras } & $\mathrm{pH}$ & $\mathrm{Ca}^{2+}+\mathrm{Mg}^{2+}$ & $\mathrm{Ca}^{2+}$ & $\mathbf{M g}^{2+}$ & $\mathbf{K}^{+}$ & $\mathrm{Na}^{+}$ & $\mathbf{A l}^{3+}$ & $\mathrm{H}+\mathrm{Al}$ & $\mathbf{P}^{+}$ & $\mathbf{K}^{+}$ & $\mathrm{Na}^{+}$ & M.O. \\
\hline & & \multicolumn{7}{|c|}{$\mathrm{cmol}_{\mathrm{c}} \mathrm{dm}^{-3}$} & \multicolumn{3}{|c|}{------mg dm-3----- } & $\%$ \\
\hline Antes & 6,90 & 8,45 & 6,12 & 2,33 & 1,01 & 0,46 & 0,10 & 1,41 & 240,18 & 394,9 & 106,16 & 3,46 \\
\hline Após & 7,04 & 9,45 & 5,65 & 3,80 & 0,63 & 0,21 & 0,05 & 1,41 & 141,07 & 247,6 & 47,23 & 3,08 \\
\hline
\end{tabular}

O pH das amostras de solo simulado antes e após a biodegradação é 6,90 e 7,04, respectivamente. É tido como bom, pois ele é um fator ambiental que, juntamente com a temperatura e a umidade, irá influenciar na presença e ação dos microrganismos no solo.

Para Chandra e Rustgi (1998) e Rosa e Carraro (1999), o pH mais favorável para o desenvolvimento dos fungos está entre 5 e 7, uma vez que é uma das condições em que os fungos produzem as enzimas necessárias para a degradação dos compósitos de polietileno com partículas de casca de arroz e oxibiodegradante orgânico.

Com relação ao teor de matéria orgânica (MO), nota-se que o solo simulado antes e após o ensaio de biodegradação apresenta 3,46\% e 3,08\% de matéria orgânica, respectivamente.

Para Sobral et al. (2015), a distribuição de frequências dos teores de matéria orgânica enquadra o solo simulado (antes e após a biodegradação) como superior a 3\%; o que indica que é um solo com alto teor de matéria orgânica, que favorece a ação de microrganismos e, consequentemente a ciclagem de nutrientes.

A disponibilidade de matéria orgânica está associada à ciclagem de nutrientes, devido à ação de microrganismos que estão presentes no solo. A microbiota é responsável pela produção de enzimas que irão decompor o material orgânico, favorecendo a disponibilidade de nutrientes como nitrogênio $(N)$, fósforo $(P)$ e enxofre $(S)$ os quais são de fundamental importância para o desenvolvimento da vegetação e, consequentemente, o solo não requer de fertilização para o desenvolvimento das culturas (WANG et al., 2015; SHAHBAZ et al., 2017; YANNI et al., 2018).

A presença de íons no solo simulado pode estar associada à ação dos microrganismos na decomposição dos compósitos e, também, da matéria orgânica, a qual apresentou uma redução na sua porcentagem, de 3,46\% para 3,08\% (LIU et al., 2018). 
A decomposição da matéria orgânica ocorre devido à ação de microrganismos, entre eles o Aspergillus niger, Penicillium spp., Trichoderma spp. e Rhizopus ssp. os quais degradam a celulose, a hemicelulose e a lignina, principais constituintes da matéria orgânica vegetal que compõe o solo simulado, resultando em íons de $\mathrm{Ca}^{2+}, \mathrm{Mg}^{2+}, \mathrm{K}^{+}, \mathrm{Na}^{+}, \mathrm{P}$ e $\mathrm{K}^{+}$os quais podem ser consumidos pelos microrganismos, em algum momento do processo metabólico, e, ainda, podem ficar no solo, para absorção pelas plantas (SHAHBAZ et al., 2017; WALMSLEY; CERDÁ, 2017).

Há um conjunto de fatores abióticos que contribuem para a presença de microrganismos no solo: pH, temperatura, teor de matéria orgânica (PRIMAVESI, 2002). Deste modo, o solo simulado reúne condições favoráveis para a presença e ação de microrganismos -Aspergillus niger, Penicillium spp., Trichoderma spp. e Rhizopus ssp.- os quais foram identificados na caracterização microbiológica do solo.

\section{CONSIDERAÇÕES FINAIS}

O compósito PEAD/CA/EG apresentou um maior grau de biodegradação após envelhecimento acelerado por radiação UV, como sugerido pelas imagens de líquido penetrante, as quais mostram fissuras, rachaduras e buracos nas superfícies dos compósitos, favorecidos pela presença do oxibiodegradante orgânico e das partículas de casca de arroz.

Pela análise microbiológica do solo, foi possível a identificação de colônias de microrganismos com atividade de biodegradação do polietileno, como Aspergillus níger, Penicillium spp., Trichoderma spp., Rhizopus spp, que degradam a superfície dos compósitos a partir da formação de biofilmes.

Portanto, é possível afirmar que o compósito PEAD/CA/EG apresentou potencialidade de uso, uma vez que apresentou características de biodegradação -graças ao aditivo oxibiodegradante orgânico- sem produzir impactos ambientais negativos ao meio ambiente e à saúde humana pelo acúmulo de metais pesados e/ou demora na degradação da matriz polimérica. 


\section{REFERÊNCIAS}

ABRUSCI C.; PABLOS, J. L.; CORRALES, T.; et al. Biodegradation of photo-degraded mulching films based on polyethylenes and stereates of calcium and iron as pro-oxidant additives. International Biodeterior \& Biodegradation, v. 65, n. 3, p. 451-459, 2011.

ABRUSCI, C.; PABLOS, J. P.; MARÍN, I.; et al. Comparative effect of metal stearates as prooxidant additives on bacterial biodegradation of thermal- and photo-degraded low density polyethylene mulching films. International Biodeterioration \& Biodegradation, v. 83, p. 2532, 2013.

ARRÁEZ, F. J.; ÁVILA, M.; ARNAL, M. L.; et al. Estudio del efecto de aditivos pro-oxidantes "oxo" en el proceso abiótico de oxodegradación de películas de polipropileno y poliestireno de alto impacto. Rev. LatinAm. Metal. Mat., v. 38, n. 1, p. 1-18, 2018.

BARNETT, H. I.; HUBTER, B. B. Illustrated genera of imperfect fungi. New York: Mac Millan Publish Company, 1972.

BENÍTEZ, A., SÁNCHEZ, J. J., ARNAL, M. L.; et al. Monitoring abiotic degradation of branched polyethylenes formulated with pro-oxidants through different mechanical tests. Polymer Degradation and Stability, v. 98, p. 1705-1716, 2013.

BHARDWAJ, H.; GUPTA, R.; TIWARI, A. Communities of microbial enzymes associated with biodegradation of plastics. Journal Polymer Environmental, v. 1, p. 16-21, 2012.

BONHOMME, S.; CUER, A.; DELORT, A-M.; et al. Environmental biodegradation of polyethylene. Polym Degrad Stab, v. 81, p. 441-452, 2003.

BOTELHO, G. et al. Enhancement of the thermooxidative degradability of polystyrene by chemical modification. Polymer Degradation and Stability, Oxford, v.86, p.493-497, 2007.

CHANDRA, R.; RUSTGI, R. Biodegradable polymers, Progress Polymer Science, v. 23, pp. 1302-1304, 1998.

CORTI, A.; MUNIYASAMY, S.; VITALI, M.; et al. Oxidation and biodegradation of polyethylene films containing pro-oxidant additives: synergistic effects of sunlight exposure, thermal aging and fungal biodegradation. Polym Degrad Stab, v. 95, n. 6, p. 11061114, 2010.

DEVI, R. S.; KANNAN, V.R.; NIVAS, D.; et al. Biodegradation of HDPE by Aspergillus spp. from marine ecosystem of Gulf of Mannar, India. Marine Pollution Bulletin, v. 96, p. 32-40, 2015.

EMBRAPA. Manual de análises químicas de solos, plantas e fertilizantes / editor técnico, Fábio Cesar da Silva. - 2. ed. rev. ampl. - Brasília, DF: Embrapa Informação Tecnológica, 2009. 
FOCKE, W. W.; MASHELE, R. P.; NHLAPO, N. S. Stabilization of low-density polyethylene films containing metal stearates as photodegradants. Journal of Vinyl \& Additive Technology, v. 17, p. 21-27, 2011.

GOMES, L. B.; KLEIN, J. M.; BRANDALISE, R. N.; et al. Study of oxo-biodegradable polyethylene degradation in simulated soil. Materials Research, v. 17, n. 1, p. 121-126, 2014.

GROSS, R. A.; KALRA, B. Biodegradable polymers for the environment. Science, v. 97, p. 803-807, 2002.

JAKUBOWICZ, I. Evaluation of degradability of biodegradable polyethylene (PE). Polymer Degradation and Stability, n. 80, p. 39-43, 2003.

JAKUBOWICZ, I.; YARAHMADI, N.; ARTHURSON, V. Kinetics of abiotic and biotic degradability of low-density polyethylene containing prodegradant additives and its effect on the growth of microbial communities. Polymer Degradation and Stability, v. 96, p. 919928, 2011.

KHABBAZ, F.; ALBERTSSON, A-C.; KARLSSON, S. Chemical and morphological changes of environmentally degradable polyethylene films exposed to thermo-oxidation. Polymer Degradation and Stability, v. 63, p. 127-138, 1999.

KYAW, B. M.; CHAMPAKALAKSHMI, R.; SAKHARKAR, M. K.; et al. Biodegradation of low density polythene (LDPE) by Pseudomonas Species. Indian J Microbiol, v. 52, n. 3, p. 411419, 2012.

KIRYAKOVA, D., MIHALEVA, S., ATANASSOV, A. Effect of metal acetylacetonates on the photooxidative destruction of high density polyethylene. Materials Research, v. 19, n. 4, p. 901-907, 2016.

KONDURI, M. K. R., KOTESWARAREDDY, G., KUMAR, D. B. R., et al. Effect of pro-oxidants on biodegradation of polyethylene (LDPE) by indigenous fungal isolate, Aspergillus oryzae. Journal of Applied Polymer Science, v. 120, p. 3536-3545, 2011.

KOUTNY, M.; SANCELME, M.; DABIN, C.; et al. Acquired biodegradability of polyethylenes containing prooxidant additives. Polym. Degrad. Stabil., v. 91, p. 1495-1503, 2006.

LACAZ, C.S.; PORTO, E.; VACCARI, E.M.H.; et al. Guia para identificação: fungos, actinomicetos e algas de interesse médico. São Paulo: Sarvier; 1998.

LIU, Y.; ZANG, H.; GE, T.; et al. Intensive fertilization (N, P, K, Ca, and S) decreases organic matter decomposition in paddy soil, Applied Soil Ecology, 2018.

MAGNA, G. A. M.; MACHADO, S. L.; PORTELLA, R. B.; et al. Avaliação da exposição ao Pb e $\mathrm{Cd}$ em crianças de $\mathrm{o}$ a 17 anos por consumo de alimentos vegetais cultivados em solos contaminados no município de Santo Amaro (BA). Eng Sanit Ambient., p. 3-12, 2014. 
MANANGAN, T.; SHAWAPHUN, S.; SANGSANSIRI, D.; et al. Nano-sized titanium dioxides as photo-catalysts in degradation of polyethylene and polypropylene packagings. Science Journal Ubon Ratchathani University, v. 1, n. 2, p. 14-20, 2010.

MARYUDI, R. M. Y.; NOUR, A. H.; BEG, H. M. D. H.; et al. The degradation of high density polyethylene containing manganese stearate under accelerated weathering. IC-GWBT2012, Ahmad Dahlan University, p. 11-20, 2012.

MARYUDI, R. M. Y.; HISYAM, A., YUNUS, R. M. N et al. Thermo-oxidative degradation of high density polyethylene containing manganese carboxylate. International Journal of Engineering Research and Applications, v. 3, n. 2, p. 1156-1165, 2013.

MONTAGNA, L. S.; FORTE, M. M. C.; SANTANA, R. M. C. Study on the Accelerated biodegradation of PP modified with an organic pro-degradant additive. J. Appl. Polym. Sci., p. 15-18, 2014.

MATHUR, G.; MATHUR, A.; PRASAD, R. Colonization and degradation of thermally oxidized high-density polyethylene by Aspergillus niger (ITCC No 6052) isolated from plastic waste dumpsite. Biorem. J., v. 15, n. 2, p. 69-76, 2011.

PETROBRÁS. Ensaio não-destrutivo - líquido penetrante. N-1596 REV. E NOV / 2003. http://ftp.demec.ufpr.br/disciplinas/TM177/Prof.Okimoto/N-1596\%5B1\%5D.pdf

PRIMAVESI, A. Manejo ecológico do solo: a agricultura em regiões tropicais. São Paulo: Nobel, 2002, 549p.

ROSA, D.S.; CARRARO, G. Avaliação de plásticos biodegradáveis sob envelhecimento acelerado em solo com diferentes $\mathrm{pH}$. In: $5^{\circ}$ Congresso Brasileiro de Polímeros, pp. 1250$1254,1999$.

SAMAL, S.K.; FERNANDES, E.G.; CORTI, A.; et al. Bio-based Polyethylene- Lignin Composites Containing a Pro-oxidant/Pro-degradant Additive: Preparation and Characterization, J Polym Environ, v. 22, pp. 58-68, 2014.

SHABANI, F.; KUMAR, L.; ESMAEILI, A. A modelling implementation of climate change on biodegradation of Low-Density Polyethylene (LDPE) by Aspergillus niger in soil. Global Ecology and Conservation, v. 4, p. 388-398, 2015.

SHAHBAZ, M.; KUZYAKOV, Y.; MUHAMMAD, S.; HEITKAMP, F.; et al. Microbial decomposition of soil organic matter is mediated by quality and quantity of crop residues: mechanisms and thresholds. Biol. Fertil. Soils, v.1, p. 1-16, 2017.

SOBRAL, L.F.; BARRETTO, M.C.V.; SILVA, A.J.; et al. Guia prática para a interpretação de resultados de análise de solo. Aracaju: EMBRAPA, 2015. 
STARK, N. M., MATUANA, L. M. Influence of photostabilizers on wood flour-HDPE composites exposed to xenon-arc radiation with and without water spray. Polymer Degradation and Stability, v. 91, p. 3048-3056, 2006.

TOKIWA, Y.; CALABIA, B. P.; UGWU, C. U.; et al. Biodegradability of plastics. International Journal of Molecular Science, v. 10, p. 3722-3742, 2009.

YAMADA-ONODERA, K.; MUKUMOTO, H.; KATSUYAYA, Y.; et al. Degradation of polyethylene by a fungus Penicillium simplicissimum YK. Polymer Degradation and Stability, v. 72, p. 323-327, 2001.

YANG, R.; LIU, Y.; YU, J.; et al. Thermal oxidation products and kinetics of polyethylene composites. Polym Degrad Stab., v. 91, n. 8, p.1651-1657, 2006.

YANNI, S. F.; DIOCHON, A.; HELGASON, B. L.; et al. Temperature response of plant residue and soil organic matter decomposition in soil from different depths. European Journal of Soil Science, v. 69, n. 2, p. 35-245, 2018.

WALMSLEY, A; CERDÁ, A. Soil macrofauna and organic matter in irrigated orchards under Mediterranean climate. Biological Agriculture \& Horticulture, v. 33, p. 247-257, 2017.

WANG, H.; BOUTTON, T.; XU, W.; et al. Quality of fresh organic matter affects priming of soil organic matter and substrate utilization patterns of microbes, Scientific Report, v. 5, pp. 1-13, 2015. 